\title{
Synthesis of Mn-doped CdTe nanoparticles and their application as fluorescence sensors
}

\author{
Li Li ${ }^{1}$, Ya-xiang Lu, Yong-Xiao Cai, Yu Cheng, Ya-ping Ding* \\ Department of Chemistry, Shanghai University, Shanghai 200444, P. R. China \\ Corresponding e-mail address: wdingyp@sina.com
}

\begin{abstract}
:
Mn-doped CdTe nanoparticles (NPs) were synthesized via a novel, facile method at low temperature. The doped NPs were directly synthesized in aqueous solution by mixing $\mathrm{CdCl}_{2} \cdot 2.5 \mathrm{H}_{2} \mathrm{O}$, fresh NaHTe solution, thioglycolic acid (TGA) and $\mathrm{MnCl}_{2} \cdot 4 \mathrm{H}_{2} \mathrm{O}$ under suitable conditions. Mn-doped CdTe NPs evaluated as fluorescence sensors for bovine serum albumin (BSA) in aqueous solution. Experiment results showed that the fluorescence emission of Mn-doped CdTe NPs was enhanced significantly by BSA, while other substances exhibited no significant effect on NPs. Based on the distinct optical properties of Mn-doped CdTe NPs with BSA, Mn-doped CdTe NPs can be developed as potential identified fluorescence sensors for determination of brovine serum albumin (BSA) in aqueous solution.
\end{abstract}

Key words: Mn-doped CdTe QDs, Fluorescence, thioglycolic acid, Sensors, bovine serum albumin (BSA)

\section{Introduction}

Semiconductor nanoparticles (or quantum dots, QDs), with their unique properties such as good optical characteristics, large surface-tovolume ratio, high electron-transfer efficiency, biocompatibility, dimensional similarities with biological macromolecules and high surface reaction activity, have experienced huge development and a great widening of the fields of applications [1-6]. As a result of tremendous progress made in the understanding of many properties of semiconductors nanoparticles, there have been considerable interests from researchers to go beyond the pure nanoparticles and investigate materials that are intentionally doped with impurities [7-14]. Doped metal nanoparticles (NPs) without doubt have been among the most exhilarating research topics in recent years. Due to quantum confinement of charge carriers in tiny spaces, NPs show some unique and fascinating properties. By ity provides a means of coupling the optical and magnetic properties of these materials via $s p-d$ exchange interaction [15]. Ma et al. [16] have reported the Mn-doped $\mathrm{ZnSe}$ QDs for the determination of L-tyrosine in enzyme catalysis system. He et al. [17] have reported the Mn-doped ZnS QDs for the detection of enoxacin in biological fluids. Much effort has been made to realize Mn doping in $\mathrm{ZnS}, \mathrm{CdS}, \mathrm{ZnSe}$ and CdSe NPs in order to produce new materials.
However, Mn-doped CdTe nanoparticles are not fully developed, despite their high potential over a range of applications. Until now, as far as we know, there is no researcher reported on the synthesis of Mn-doped CdTe NPs and their application as fluorescence sensors for determination bovine serum albumin (BSA). A new method for the determination of BSA has been developed based on enhancement of the fluorescence of $\mathrm{Mn}$-doped $\mathrm{CdTe}$ quantum dots by BSA in aqueous solution.

\section{Experimental}

\section{Synthesis of CdTe NPs}

NaHTe solution was prepared using a modified literature procedure by treatment of $\mathrm{Te}$ powder with excess $\mathrm{NaBH}_{4}$ at a molar ratio of 1:20 [18]. Sodium borohydride was added to a small flask with $5 \mathrm{~mL}$ doubly distilled water for dissolving and connected to the nitrogen to drive off the oxygen dissolving in water. Then, adding Te powder into the oxygen-free water. During the course of the reaction, a small outlet connected to the flask was kept open to discharge the pressure from the resulting $\mathrm{H}_{2}$. The NaHTe solution was obtained when the color of the solution changed to transparent (Te powder is completely dissolved.) 


\section{Synthesis of TGA-capped CdTe nanoparticles}

The synthesis of the CdTe NPs was at the molar ratio 1:0.5:2.4 of $\mathrm{Cd}^{2+} / \mathrm{Te}^{2-} / \mathrm{TGA}$ in our experiments. In a three-necked round-bottomed flask, $2 \mathrm{mmol}$ of $\mathrm{CdCl}_{2} \cdot 2.5 \mathrm{H}_{2} \mathrm{O}$ was dissolved with $200 \mathrm{~mL}$ doubly distilled water, and added $4.8 \mathrm{mmol}$ of TGA under stirring. Then adjusted the $\mathrm{pH}$ of the solution to 6 by the addition of 1.0 $\mathrm{mol} / \mathrm{L} \mathrm{NaOH}$ solution dropwise. The resulted solution was drive off dissolved $\mathrm{O}_{2}$ in the solution by $\mathrm{N}_{2}$ bubbling for $30 \mathrm{~min}$. Under vigorous stirring, oxygen-free $\mathrm{NaHTe}$ was injected into the above solution. The reaction between $\mathrm{Cd}^{2+}$ and NaHTe took place immediately upon the injection of NaHTe solution. The colorless solution changed to golden yellow.

\section{Synthesis of Mn-doped CdTe nanoparticles}

Mn-doped CdTe nanoparticles (NPs) were directly synthesized in aqueous solution by mixing TGA-capped CdTe nanoparticles, $0.01 \mathrm{mM} \mathrm{MnCl} \mathrm{M}_{2} \cdot 4 \mathrm{H}_{2} \mathrm{O}$ under suitable conditions. After boiling the solution for $8 \mathrm{~min}$, the color of solution changed to orange. Keep the solution at $60^{\circ} \mathrm{C}$ for 40 min to obtain the Mn-doped CdTe NPs with long fluorescence lifetime.

\section{Results and discussion}

\section{The comparison of optical properties}

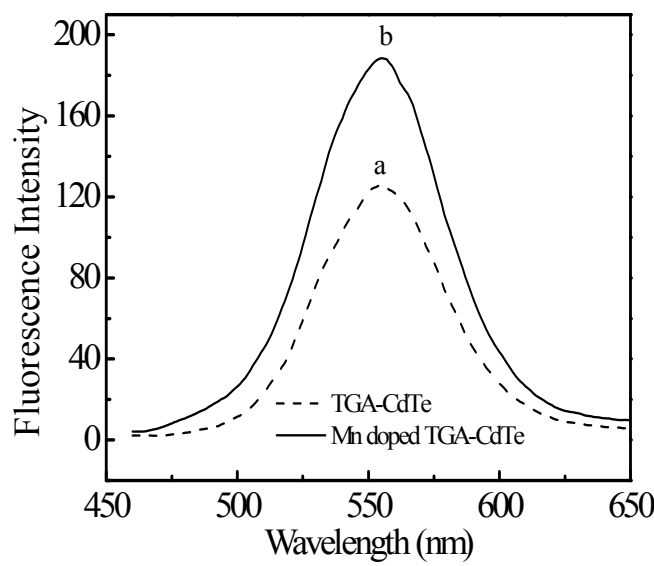

Fig. 1: Fluorescence emission spectra of (a) TGACdTe nanoparticles and (b) Mn-doped TGA-CdTe nanoparticles with excitation wavelength at $351 \mathrm{~nm}$.

The optical properties of Mn-doped TGACdTe NPs and undoped NPs were characterized by using fluorescence spectroscopy (Fig. 1). The emission peaks both center at $555 \mathrm{~nm}$, and the spectra are characterized by good symmetry and relatively narrow spectral width. The most striking evidence for the doping is the enhancement in the emission intensity of the Mn-doped CdTe in relation to the pure CdTe nanoparticles, which will be helpful for further research.

\section{Characterization by XRD}

The XRD pattern (Fig. 2) was obtained from Mn-doped TGA-CdTe powders precipitated from an aqueous solution of Mn-doped CdTe NPs with excess isopropanol. The XRD spectrum which scans over the 2 theta $(\theta)$ range of $10-80^{\circ}$ shows diffractive peaks at $25.43^{\circ}$ and $42.89^{\circ}$, corresponding to the crystal planes 101 and 103, confirming that it has a hexagonal wurtzite crystalline structure. According to the Debye-Scherrer equation, $D=0.89 \lambda / \beta \cos \theta$, the average size of $M n$-doped TGA-CdTe NPs is $23.746 \mathrm{~nm}$.

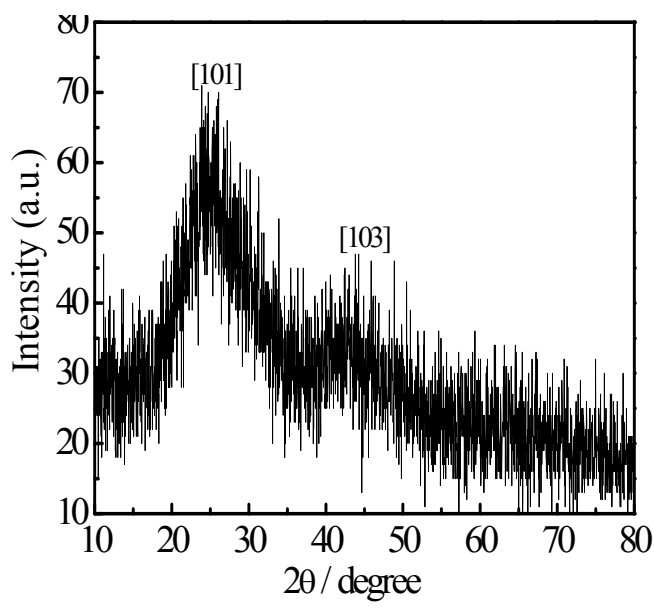

Fig.2: XRD pattern of the as-prepared Mn-doped $T G A-C d T e$ nanoparticles.

\section{Characterization by TEM}

In order to investigate the morphology and size of the prepared nanoparticles, TEM measurement was carried out (Fig. 3). As shown in Fig. 3, Mn-doped CdTe nanoparticles were nearly monodisperse spherical in shape with an average particle size about $50 \mathrm{~nm}$, which was a little bigger than the size estimated by the Scherrer formula calculations based on XRD pattern.

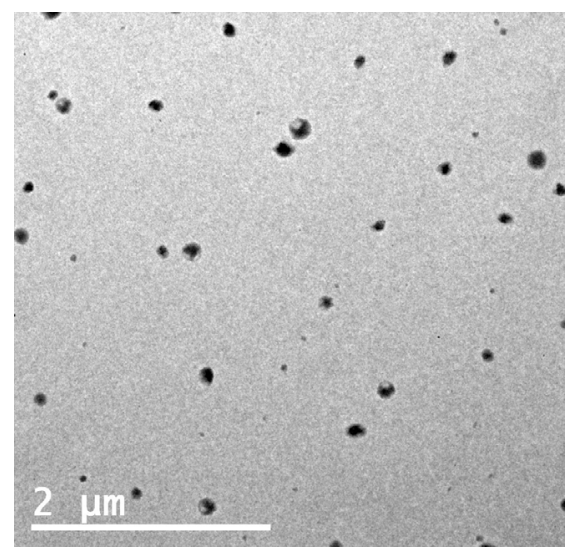

Fig.3: TEM images of Mn-doped CdSe. 


\section{Influence of $\mathrm{pH}$}

The influence of $\mathrm{pH}$ on the fluorescence intensity of Mn-doped CdTe system in the absence and presence of BSA was shown in Fig. 4. The low fluorescence intensity in acidic medium is the result of dissociation of the nanoparticles due to protonation of the surface dinding thiolates [19]. With the increase of $\mathrm{pH}$, the deprotonation of the thiol group in the thioglycolic acid molecule occurs. This deprotonation could strengthen the covalent bond between $\mathrm{Cd}$ and thioglycolic acid molecule, which bring about the fluorescence intensity enhance with $\mathrm{pH}$ increasing. Besides, alkali medium is suitable for both pure $\mathrm{Mn}$ doped CdTe and the presence of BSA. However, the fluorescence intensity begins to decline with the further increase of $\mathrm{pH}$ value. Therefore, the exploration of the most suitable $\mathrm{pH}$ value is ongoing.



Fig. 4. Effect of $\mathrm{pH}$ on the fluorescence intensity of Mn-doped CdTe system in the absence and presence of BSA.

\section{Feasibility of the Mn-doped CdTe QDs for the detection of BSA.}



Fig. 5. BSA concentration-dependent emission of the Synthesized water-soluble Mn-doped CdTe QDs.
To explore the potential of the Mn-doped CdTe QDs for the detection of BSA, we investigated the effect of the Mn-doped CdTe QDs in the presence of different concentrations of BSA. Spectroscopic measurements were performed in aqueous solution. Fig. 5 shows the enhancement response of the Mn-doped CdTe QDs to BSA in aqueous solution. The addition of BSA enhanced the fluorescence intensity of Mn-doped CdTe QDs.

\section{Acknowledgements}

This work was supported by the National Natural Science Foundation of China (No. 20975066, No. 41140031), the NanoFoundation of Science and Techniques Commission of Shanghai Municipality (No. 0952nm01500), the Leading Academic Discipline Project of Shanghai Municipal Education Commission (No. J50102), and the Graduate Innovation Foundation of Shanghai University (SHUCX112027).

\section{References}

[1] H. Bönnemann, R. M. Richards, Nanoscopic metal particlessynthetic methods and potential applications, Eur J Inorg Chem 10:24552480(2001); doi: 10.1002/1099-0682(200109)

[2] C. N. R. Rao, G. U. Kulkarni, P. J. Thomas, P. P. Edwards, Metal nanoparticles and their assemblies, Chem Soc Rev 29,27-35(2000); doi: 10.1039/A904518J

[3] A. J. Bard, Ding Z, N. Myung, Electrochemistry and electrogenerated chemiluminescence of semiconductor nanocrystals in solutions and in films, Struct Bond 118,1-57(2005); doi: $10.1007 / \mathrm{b} 137239$

[4] P. Alivisatos, The use of nanocrystals in biological detection, Nat Biotechnol 22,4752(2004); doi:10.1038/nbt927

[5] I. L. Medintz, H. T. Uyeda, E. R. Goldman, H . Mattoussi, Quantum dots bioconjugates for imaging, labelling and sensing, Nat Mater 6,435446(2005); doi:10.1038/nmat1390

[6] J. Riegler, P. Nick, U. Kielmann, T. Nann, Visualizing the self-assembly of tubulin with luminescent nanorods, J Nanosci Nanotech 5,380-385(2003); doi: org/10.1166/jnn

[7] L. Levy, J. F. Hochepied, M. P. Pileni, Control of the Size and Composition of Three Dimensionally Diluted Magnetic Semiconductor Clusters, J Phys Chem 100,18322- $18326 \quad$ (1996); doi: $10.1021 / j p 960824 \mathrm{w}$

[8] F. V. Mikulec, M. Kuno, M. Bennati, D. A. Hall, R. G. Griffin, M. G. Bawendi, Organometallic Synthesis and Spectroscopic Characterization of Manganese-Doped CdSe Nanocrystals, J Am Chem Soc 122, 2532-2540 (2000); doi: $10.1021 / j a 991249 n$ 
[9] M. A. Malik, P. O'Brien, N. Revaprasadu, Synthesis of TOPO-capped Mn-doped $\mathrm{ZnS}$ and CdS quantum dots, J Mater Chem 11,2382(2001); doi: $10.1039 / \mathrm{b} 102709 \mathrm{n}$

[10] D. J. Norris, N. Yao, F. T. Charnock, T. A. Kennedy, High-Quality Manganese-Doped ZnSe Nanocrystals, Nano Lett 1,3-7,(2001); doi: $10.1021 / \mathrm{nl005503h}$

[11] K. M. Hanif, R. W. Meulenberg, G. F. Strouse, Magnetic Ordering in Doped $\mathrm{Cd}_{1-x} \mathrm{Co}_{x}$ Se Diluted Magnetic Quantum Dots, J Am Chem Soc 124,11495(2002); doi: 10.1021/ja0262840

[12] C. A. Stowell, R. J. Wiacek, A. E. Saunders, B. A. Korgel, Synthesis and Characterization of Dilute Magnetic Semiconductor Manganese-Doped Indium Arsenide Nanocrystals, Nano Lett 3, 1441-1447 (2003); doi: 10.1021/nl034419+

[13] S. C. Erwin, L. J. Zu, M. I. Haftel, A. L. Efros, T. A. Kennedy, D. J. Norris, Doping semiconductor nanocrystals, Nature 436,91-94(2005); doi:10.1038/nature03832

[14] W. C. Kwak, Y. M. Sung, T. G. Kim, W. S. Chae , Synthesis of Mn-doped zinc blende CdSe nanocrystals, Appl Phys Lett 90,173111(2007); doi.org/10.1063/1.2731682

[15] O. S. Oluwafemi, N. Revaprasadu, O. O. Adeyemi, A new synthesis of hexadecylaminecapped Mn-doped wurtzite CdSe nanoparticles, Materials Letters 64, 1513-1516 (2010); doi: 10.1016/j.matlet.2010.04.007

[16] Q. Ma, W. Yu, H. Huang, X. Su, Determination of L-tyrosine based on luminescence quenching of Mn-doped ZnSe quantum dots in enzyme catalysis system, Journal of Fluorescence 21, 125-131 (2011); doi: 10.1007/s10895-010-0696-z

[17] Y. He, H. F. Wang, X. P. Yan*, Exploring MnDoped ZnS Quantum Dots for the RoomTemperature Phosphorescence Detection of Enoxacin in Biological Fluids Anal, Chem. 80, 3832-3837(2008); doi:10.1021/ac800100y

[18] W. F. Zhao, Y. S. Fung, W. S. O, M. P. L. Cheung, L-Cysteine-capped CdTe Quantum Dots as a Fluorescence Probe for Determination of Cardiolipin, Anal. Sci. 26, 879(2010); doi:10.2116/analsci.26.879

[19] M. Gao, S. Kirstein, H. Mohwald, A.L. Rogach, A. Kornowski, A. Eychmuller, H. Weller, Strongly photoluminescent CdTe nanocrystals by proper surface modification, The Journal of Physical Chemistry B 102, 8360-8363(1998); doi: $10.1021 / \mathrm{jp} 9823603$ 